\title{
GEOMETRIC THEORY OF WEAK KOROVKIN SETS
}

\author{
M. N. N. NAMBOODIRI
}

Abstract. A set $S$ of generators of an abstract $W^{*}$-algebra $\mathscr{A}$ is called weakly hyperrigid if for every faithful representation of $\mathscr{A}$ on a Hilbert space $\mathscr{A} \subseteq B(H)$ and every net $\left\{\Phi_{\alpha}\right\}_{\alpha \in I}$ of completely positive maps, of norm $\leqslant 1$, on $B(H)$,

$$
\lim _{\alpha} \Phi_{\alpha}(s)=s \text { weakly, for all } s \in S \Rightarrow \lim _{\alpha} \Phi_{\alpha}(a)=a \text { weakly },
$$

for all $a \in \mathscr{A}$. This is analogous to W. B. Arveson's [6] hyperrigid sets in $C^{*}$ algebras. A characterisation of weakly hyperrigid sets and a noncommutative analogue of Y. Saskin's theorem [17] on geometric characterisation of Korovkin sets in $C(X)$ is proved.

Mathematics subject classification (2010): Primary 46L07; Secondary 46L52. Keywords and phrases: Korovkin, $W^{*}$-algebras, completely positive maps.

\section{REFERENCES}

[1] F. Altomare And M. CAmpiti, Korovkin type approximation theory and its applications, de Gruyter Studies in Mathmatics, Berlin, New York, 1994.

[2] F. Altomare, Korovkin-type theorems and approximation by positive linear operators, Surveys in Approximation Theory 5 (2010) 92-164.

[3] W. B. Arveson, Subalgebras of $C^{*}$-algebras, Acta. Math. 123 (1969), 141-224.

[4] W. B. ARveson, Subalgebras of $C^{*}$-algebras II, Acta. Math. 128 (1972), 271-308.

[5] W. B. ARVESON, The noncommutative Choquet boundary I, arXIV:MATH0701329 V4 1 [math OA], $19 \mathrm{Feb} 2007$.

[6] W. B. ARVESON, The noncommutative Choquet boundary II: Hyperrigidity, arXIV:08102751 V4 [math OA], 28 May 2009.

[7] H. Berens And G. G. LorentZ, Geometric theory of Korovkin sets, J. Approx. Theory, Acad. Press 1975.

[8] P. P. Korovkin, Linear operators and approximation theory, Hindustan Publ. Corp. Delhi, India, 1960.

[9] B. V. Limaye AND M. N. N. NAmBoodiri, Korovkin-type approximation on $C^{*}$ algebras, J. Approx. Theory 34, 3 (1982), 237-246.

[10] B. V. Limaye AND M. N. N. NAmboodiri, Weak Korovkin approximation by completely positive maps on $B[H]$, J. Approx. Theory, Acad. Press, 1984.

[11] B. V. LimAYE AND M. N. N. NAMBoodiRI, A generalized noncommutative Korovkin theorem and closedness of certain sets of convergence, Ill. J. Math 28 (1984), 267-280.

[12] M. N. N. NAmBoodiRI, Geometric formulation of noncommutative (weak) Korovkin sets, Short talk given at ICM Satellite Conference on Functional Analysis and Operator Theory held at ISI Bangalore, August 07-10, 2010.

[13] Ola Brattele and D. W. Robinson, Operator algebras and quantum statistical mechanics-I, C* and $W^{*}$ algebras, Symmetry groups, Decomposition of states, Springer-Verlag, New York-HeidelbergBerlin, 1979.

[14] W. M. Priestley, A noncommutative Korovkin theorem, J. Approx. Theory 16, 3 (1976), 251-260.

[15] A. G. Robertson, A Korovkin theorem for Schwartz maps on $C^{*}$ algebras, Math. Z. 156, 2 (1977), 205-207. 
[16] A. G. Robertson, Assymmetric invariants sets for completely positive maps on $C^{*}$-star algebras, Bull. Austral. Math. Soc. 33, 3 (1986), 471-473.

[17] Y. A. SASKIN, Korovkin systems in spaces of continuous functions, A. M. S. Translations [2] 54 (1966), 125-144.

[18] S. TAKAHASI, Korovkin's theorem for $C^{*}$-algebras, J. Approx. Theory 27 (1979).

[19] M. UCHIYAMA, Korovkin type theorems for Schwartz maps and operator monotone functions in $C^{*}$ algebras, Math. Z. 230 (1999).

[20] D. E. Wulbert, Convergence of operators and Korovkin's theorem, J. Approximation Theory 1 (1968), 381-390. 